\title{
Supercontinuum Generation using Mode-Locked picoseconds Pulse Laser
}

\author{
S. W. Harun* \\ Department of Electrical Engineering, University of Malaya, 50603 Kuala Lumpur, Malaysia \\ M. R. A. Moghaddam and H. Ahmad \\ Photonics Research Center, University of Malaya, 50603 Kuala Lumpur, Malaysia.
}

\begin{abstract}
Supercontinuum (SC) generations in two nonlinear fibers are demonstrated using a passively mode-locked Erbium-doped fiber laser (EDFL). The EDFL is constructed using a semiconductor saturable absorber to generate 1.3 ps pulses with average power of $4.9 \mathrm{~mW}$ and repetition rate of $9.9 \mathrm{MHz}$ at $1564 \mathrm{~nm}$. The picosecond pulses are amplified by an Erbium-doped fiber amplifier to achieve an average output power of $\approx 13 \mathrm{dBm}$ before it is launched into a 50m long photonic crystal fiber (PCF) and $100 \mathrm{~m}$ long highly nonlinear fiber (HNLF) for SC generation. Continuums starting from $1210 \mathrm{~nm}$ and $1370 \mathrm{~nm}$ for PCF and HNLF, respectively up to wavelength region of more than $1750 \mathrm{~nm}$ are achieved with average power of $\approx 10 \mathrm{dBm}$, which corresponds to $50 \%$ power conversion efficiency from pump to continuum.
\end{abstract}

KEYWORDS: Supercontinuum generation, mode-locked fiber laser, picoseconds fiber laser, nonlinear effect.

\section{INTRODUCTION}

Fibers and semiconductors of optical wavelengths beyond one micron have various applications in bio-medicine, chemical sensing, semiconductor processing, and communication networks and systems. In fiber optic communications, various methods of increasing the amount of signal that can be carried on a single fiber have been explored. For broadband dense wavelength division multiplexing (DWDM), multiwavelength continuous wave $(\mathrm{CW})$ source is required. This source can be generated using fiber-based supercontinuum and spectrum-slicing of its longitudinal modes [1]. Supercontinuum (SC) generation is a complex nonlinear phenomenon that is characterized by the dramatic spectral broadening of intense light pulses passing through a nonlinear material. It is also currently a subject of intense research for applications in the fields of optical frequency metrology, generation of ultrashort pulses and biomedical optics. and wavelength division multiplexing (WDM) optical communication systems [2-3].

Motivated by important applications in precision frequency metrology, initial research efforts on this topic have focused on developing a clear physical understanding of the underlying mechanisms and noise properties of SC generation seeded by femto-second pump pulses, a regime which is now very well-understood [5-6]. Research is now shifting towards detailed studies of the supercontinuum generation using picosecond to nanosecond pulses, or even a continuous wave pump where spectral broadening is initiated in the so-called "long pulse" regime [7]. In this paper, an SC generation is demonstrated using amplified picoseconds pump pulses in a highly

*E-MAIL: swharun@um. edu.my nonlinear fiber. The picoseconds pulses are generated using an all-fiber passively mode locked Erbium-doped fiber laser. The dominant nonlinear effects responsible for the continuum generation are stimulated Raman scattering, four-wave mixing, self-phase modulation, solitons and etc.

\section{EXPERIMENT}

SC generation experiments are performed for two different nonlinear fibers; a $50 \mathrm{~m}$ long photonic crystal fiber (PCF) and a $100 \mathrm{~m}$ long highly non-linear fiber (HNLF). The experimental set-up for the system is illustrated in Fig. 1, which consists of a pulse oscillator, an Erbium-doped fiber amplifier (EDFA) and a piece of highly nonlinear fiber. A modelocked ring Erbium-doped fiber laser (EDFL) is used as the oscillator, which consists of a piece of EDF, a WDM coupler, an isolator, a saturable absorber, a polarizing beam splitter (PBS), two polarization controllers and an output coupler. The EDFL cavity consists of three different fibers that are a $4 \mathrm{~m}$ long EDF (with a group-velocity dispersion (GVD) of $+23.51 \pm 0.21 \mathrm{ps}^{2} / \mathrm{km}$ at $\lambda=1545 \mathrm{~nm}$ ), a $5.58 \mathrm{~m}$ long SMF-28 which is used in the output coupler and two PCs (with a GVD of $-22.75 \pm 0.91 \mathrm{ps}^{2} / \mathrm{km}$ at $1545 \mathrm{~nm}$ ) and a $0.42 \mathrm{~m}$ long SMF of $980 / 1550 \mathrm{~nm}$ WDM coupler (with a GVD of $-9.94 \pm 0.18$ $\mathrm{ps}^{2} / \mathrm{km}$ at $\left.1548 \mathrm{~nm}\right)$. The EDF is backward pumped by a $980 \mathrm{~nm}$ laser diode via the WDM. The EDF used has a mode field diameter of $5.9 \mu \mathrm{m}$ with the maximum peak absorption of around $11.8 \mathrm{~dB} / \mathrm{m}$ at $978 \mathrm{~nm}$. A semiconductor saturable absorber is used for the initiation and stabilization of modelocking at around $1550 \mathrm{~nm}$ region. The saturable absorber is a transmission type which is anti-resonant and therefore the dispersion influence is negligible in this device. It has a relaxation time constant of around $2 \mathrm{ps}$, a non-saturable loss of 


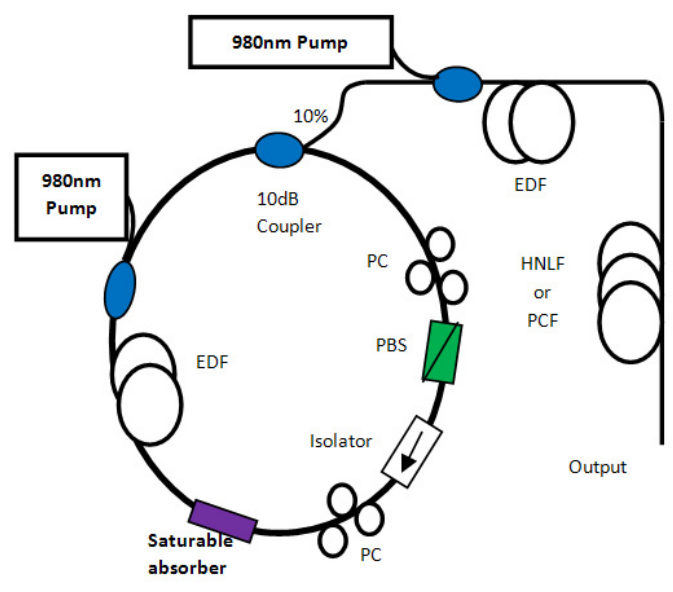

FIG. 1: Experimental set-up of the proposed system for supercontinuum generation

$10 \%$, a saturation fluence of $300 \mu \mathrm{j} / \mathrm{cm}^{2}$ and this device is designed for an operation at the central wavelength of $1550 \mathrm{~nm}$. The PC is used to rotate the polarization state and allows continuous adjustment of the birefringence within the cavity to balance the gain and loss for laser pulse generation.

The output pulses from the mode-locked EDFL are amplified by a forward pumped EDFA to achieve an output power of about $13 \mathrm{dBm}$. The amplified pulse output at $1564 \mathrm{~nm}$ is then spliced to the highly nonlinear fiber to achieve the desired spectral broadening. The PCF used in the experiment has a zero and $-0.5 \mathrm{ps} /(\mathrm{km} . \mathrm{nm})$ dispersion at wavelength of 1550 $\mathrm{nm}$ and $1561 \mathrm{~nm}$ respectively. The nonlinearity coefficient of the PCF is around $11 \mathrm{~W}^{-1} \mathrm{~km}^{-1}$. The HNLF has a zero dispersion wavelength at $1531 \mathrm{~nm}$ and a dispersion slope of 0.01 $\mathrm{ps} /\left(\mathrm{km} . \mathrm{nm}^{2}\right)$ at $1550 \mathrm{~nm}$. With effective area of $11.7 \mu \mathrm{m}^{2}$, the HNLF has a nonlinear coefficient of $11.5 \mathrm{~W}^{-1} \mathrm{~km}^{-1}$. The output power and spectrum are measured by a power meter and an optical spectrum analyzer (OSA), respectively. The entire experimental setup is fusion-spliced together.

\section{RESULT AND DISCUSSION}

The pulse characteristic of the mode locked EDFL after the $10 \mathrm{~dB}$ coupler is investigated using both an optical power and an autocorrelator in conjunction with oscilloscope. Continued wave operation starts at $\approx 11 \mathrm{~mW} 980 \mathrm{~nm}$ pump power threshold. Self-starting single pulse mode-locking is observed at $\approx 20 \mathrm{~mW}$. In the experiment, the pump power is fixed at 120 $\mathrm{mW}$. Fig. 2 shows a plot of a typical output SHG autocorrelation trace, together with the Gaussian and $\operatorname{sech}^{2}$ fits. As shown in the figure, both $\operatorname{sech}^{2}$ and Gaussian pulse profiles fit well with our experimental data. The autocorrelation fullwave half maxima (FWHM) is $\approx 1.8$ ps. Assuming a Gaussian profile, the data de-convolution gives a pulse duration of $\approx 1.3$ ps. Fig. 3 shows a measured satellite-free oscilloscope trace, which shows a stable pulse train without multiple pulsing of the laser. The repetition rate is measured to be $\approx 9.9 \mathrm{MHz}$. The

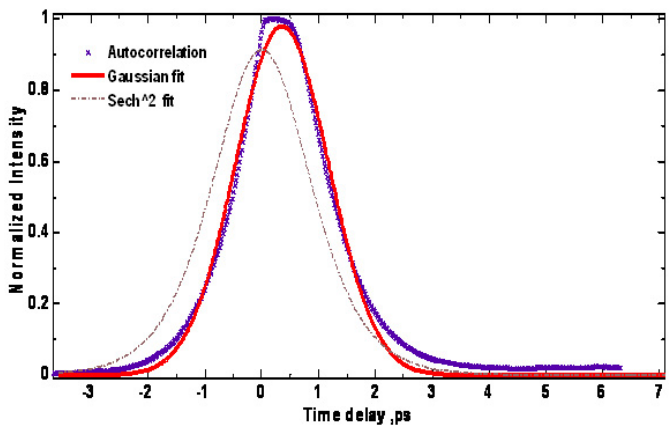

FIG. 2: Autocorrelation trace of the $1564 \mathrm{~nm}$ pulses with Gaussian and sech 2 fits. The autocorrelation signal is offset from zero for ease of viewing.

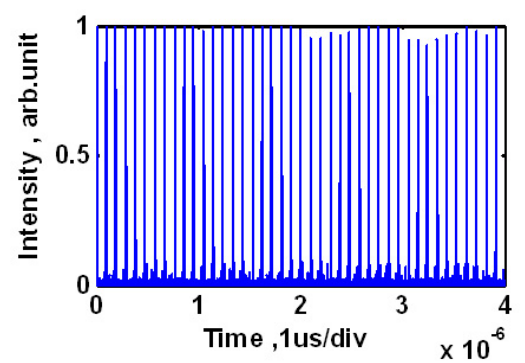

FIG. 3: An oscilloscope trace of the pulse train.

average output power is also measured to be around $4.9 \mathrm{~mW}$ using a power meter. The picosecond pulses are amplified by the EDFA to achieve an average output power of $\approx 13 \mathrm{dBm}$ before it is launched into a piece of fiber for SC generation.

Fig. 4 shows the spectra of continuum generated in both PCF and HNLF, which is pumped by a $1564 \mathrm{~nm}$ picoseconds pulse at average pump power of $13 \mathrm{dBm}$. The average power in the continuum is measured to be $? 10 \mathrm{dBm}$ using a power meter, thus, achieving a $\approx 50 \%$ power conversion efficiency from pump to continuum. As shown in Fig. 4, we observe a continuum starting from $1210 \mathrm{~nm}$ and $1370 \mathrm{~nm}$ for PCF and HNLF, respectively up to wavelength region of more than $1750 \mathrm{~nm}$. The spectral broadening of this region has not been examined due to spectral sensitivity of the OSA.

It has been reported in [8] that at the initial stage of beam propagation in fiber, symmetrical sidebands are generated due to modulation instability. With further propagation, multiple sidebands grow from the phase matched four-wave mixing (FWM) process, but only the spreading of continuum to longer wavelength side occurs due to overlap with Raman bandwidth. The spectral peak around $1564 \mathrm{~nm}$ is the EDFL pump. For the PCF, the spectrum obtained is broader compared to that of the HNLF due to the zero dispersion wavelength which is nearer to the pump wavelength. The result indicates that the nonlinear processes responsible for the wide spectrum generation occur within the first few metres of the fiber. Further propagation through longer lengths merely attenuates the spectrum due to the fiber loss. Thus, obtaining the broadest spectrum requires the shorter fiber length with 


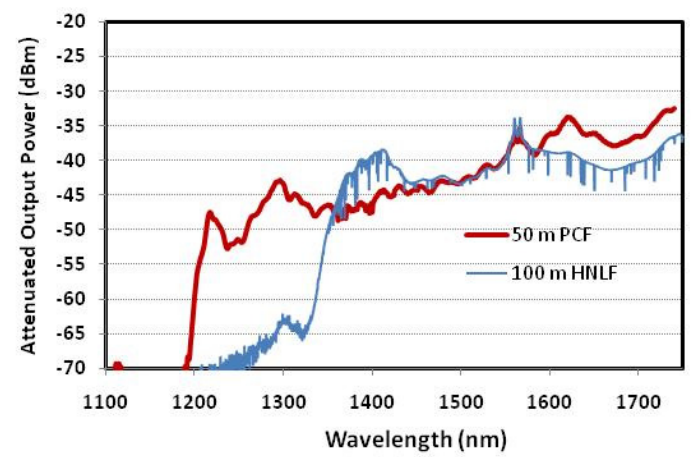

FIG. 4: Spectra of continuum generated in PCF and HNLF using picoseconds pulses at $1564 \mathrm{~nm}$ as a pump.

higher nonlinearity. The use of a longer HNLF is seen to produce a flat and homogeneous continuum in wavelength range extending from $1380 \mathrm{~nm}$ up to at least $1750 \mathrm{~nm}$ with output power variation of less than $8 \mathrm{~dB}$.

\section{CONCLUSION}

SC generations in two nonlinear fibers are demonstrated using a passively mode-locked EDFL. The EDFL is constructed using a semiconductor saturable absorber to generate $1.3 \mathrm{ps}$ pulses with average power of $4.9 \mathrm{~mW}$ and repetition rate of $9.9 \mathrm{MHz}$ at $1564 \mathrm{~nm}$. The picosecond pulses are amplified by an Erbium-doped fiber amplifier to achieve an average output power of $\approx 13 \mathrm{dBm}$ before it is launched into a $50 \mathrm{~m}$ long PCF and $100 \mathrm{~m}$ long HNLF for SC generation. Continuums starting from $1210 \mathrm{~nm}$ and $1370 \mathrm{~nm}$ for PCF and HNLF, respectively up to wavelength region of more than $1750 \mathrm{~nm}$ are achieved with average power of $\approx 10 \mathrm{dBm}$.
[1] Diddams, S. A., et. al, Phys. Rev. Lett. 84, p.5102-5105 (2000).

[2] Husakou, A.V. and Herrmann, J., Phys. Rev. Lett., 87, p.203901203904 (2002).

[3] Dunsby, C., et. al, J. Phys. D, 37, p.3296-3303 (2004).

[4] Morioka, T., et. al., Electron. Lett. 32, p.906-907 (1996).

[5] Hori, T. , et. al., J. Opt. Soc. Amer. B 21, p.1969-1980 (2004).
[6] Corwin, K. L., et. al., Phys. Rev. Lett. 90, p.113 904/1-4 (2003).

[7] Ciburys, A., et. al., Lithuanian Journal of Physics 50(1), p.83-88 (2010).

[8] Raikkonen, E., et. al, Opt. Express 14(17), p.7914 (2006). 